\title{
O FELDSPATO INDUSTRIAL DE CORONEL MURTA, MG EA PERSPECTIVA DE APLICAÇÕES À INDÚSTRIA CERÂMICA E VIDREIRA
}

\author{
SERGIO TÚLIO DE PINHO-TAVARES ${ }^{1}$, CRISTIANE CASTAÑEDA ${ }^{1} \&$ \\ ANTÔNIO CARLOS PEDROSA-SOARES ${ }^{1,2}$
}

\begin{abstract}
Resumo Foram realizadas avaliações mineralógicas e tecnológicas de minério feldspático, com base na caracterização do feldspato potássico de 21 lavras de pegmatitos derivados de granitos tipo S da suíte G4 (520-500 Ma), município de Coronel Murta, Médio Jequitinhonha, Minas Gerais. Os pegmatitos foram caracterizados utilizando ficha de dados com 13 itens, envolvendo dados gerais da lavra, geologia, além de aspectos geoambientais e de condições de exploração. Os resultados dos ensaios cerâmicos mostraram que 71 das 72 amostras submetidas ao teste do botom foram consideradas minérios de primeira qualidade para a indústria cerâmica, enquanto o teste do azulejo aprovou nove das doze amostras avaliadas. As amostras pulverizadas contendo mica e/ou caulim mostraram teores relativamente baixos em sílica $\left(\mathrm{SiO}_{2}<64 \%\right)$ e altos em alumina $\left(\mathrm{Al}_{2} \mathrm{O}_{3}>17 \%\right)$. O caulim confere ao feldspato pulverizado colorações amareladas e róseas. A amostragem sistemática por zona interna do pegmatito da lavra da Água Santa, principal produtor de feldspato do município, mostrou que este mineral na porção sudeste do corpo pegmatítico é mais rico em $\mathrm{SiO}_{2}, \mathrm{Na}_{2} \mathrm{O}$ e $\mathrm{Na}_{2} \mathrm{O}+\mathrm{K}_{2} \mathrm{O}$, enquanto a parte noroeste do pegmatito apresenta feldspato com maiores teores de $\mathrm{Al}_{2} \mathrm{O}_{3}$ e $\mathrm{Fe}_{2} \mathrm{O}_{3}$. Simultaneamente, os dados de $\mathrm{SiO}_{2}, \mathrm{Al}_{2} \mathrm{O}_{3} \mathrm{e}$ $\mathrm{Fe}_{2} \mathrm{O}_{3}$ indicam que a porção sudeste deste pegmatito é mais adequada para a produçẫo de vidro $\mathrm{B}$, enquanto a zona intermédia interna a intermédia externa NW é adequada para cerâmica de primeira qualidade. Com base na avaliação positiva do teste do botom, sobre todas as amostras, este trabalho aponta novas especificações químicas (em porcentagens-peso) para o feldspato potássico pegmatítico de Coronel Murta, que são: $\mathrm{SiO}_{2} \leq 67 ; \mathrm{TiO}_{2}<0,2 ; \mathrm{Al}_{2} \mathrm{O}_{3}>17,0 ; \mathrm{Fe}_{2} \mathrm{O}_{3} \leq 0,15 ; \mathrm{MgO} \leq 0,3 ; \mathrm{CaO} \leq 0,3 ; \mathrm{Na}_{2} \mathrm{O} 2,0-3,5 ; \mathrm{K}_{2} \mathrm{O} \geq 12,0 ; \mathrm{Perda}$ ao Fogo $<0,7 ; \mathrm{Na}_{2} \mathrm{O}+\mathrm{K}_{2} \mathrm{O} \geq 14,0$.
\end{abstract}

Palavras chaves: feldspato, cerâmica, minerais industriais.

\begin{abstract}
INDUSTRIAL FELDSPAR FROM CORONEL MURTA, MINAS GERAIS STATE, BRAZIL: APPLICATIONS FOR CERAMIC AND GLASS INDUSTRY. Mineralogical and technological evaluations of K-feldspar were accomplished from 21 pegmatites bodies, which are derived from type $S$ granites of G4 Suite (520-500 Ma), Coronel Murta, Minas Gerais. The pegmatites are characterized by using 13 items including data of deposits, geology, up to environmental aspects and exploration conditions. The results of the ceramic tests showed that 71 of the 72 samples subjected to the botom test were considered as the first quality for the ceramic industry, while the tile test approved nine of the twelve samples. The powdered samples with mica and kaolin showed relatively low contents of silica $\left(\mathrm{SiO}_{2}<64 \%\right)$ and high contents of alumina $\left(\mathrm{Al}_{2} \mathrm{O}_{3}>17 \%\right)$. The kaolin displays yellowish and reddish pink colorations to the pulverized feldspar. The systematic sampling at the internal zone of the Água Santa pegmatite, the main producer of feldspar from Coronel Murta; showed that this mineral is richer in $\mathrm{SiO}_{2}, \mathrm{Na}_{2} \mathrm{O}$ and $\mathrm{Na}_{2} \mathrm{O}+\mathrm{K}_{2} \mathrm{O}$ at the southeast portion of the pegmatite, while the northwest part of the body presents feldspar with higher contents of $\mathrm{Al}_{2} \mathrm{O}_{3}$ and $\mathrm{Fe}_{2} \mathrm{O}_{3}$. Simultaneously, the values of $\mathrm{SiO}_{2}, \mathrm{Al}_{2} \mathrm{O}_{3}$ and $\mathrm{Fe}_{2} \mathrm{O}_{3}$ indicate that the southeast portion of this pegmatite is more suitable for the production of glass type $\mathrm{B}$, while the intermediate zone can be used for ceramic of first quality. On the basis of the positive evaluation of all samples by botom test, this paper presents new chemical specifications for the $\mathrm{K}$-feldspar from Coronel Murta, that are (in weight-\%): $\mathrm{SiO}_{2} \leq 67 ; \mathrm{TiO}_{2}<0,2 ; \mathrm{Al}_{2} \mathrm{O}_{3}>17,0$; $\mathrm{Fe}_{2} \mathrm{O}_{3} \leq 0,15 ; \mathrm{MgO} \leq 0,3 ; \mathrm{CaO} \leq 0,3 ; \mathrm{Na}_{2} \mathrm{O} 2,0-3,5 ; \mathrm{K}_{2} \mathrm{O} \geq 12,0 ;$ on ignition loss $<0,7 ; \mathrm{Na}_{2} \mathrm{O}+\mathrm{K}_{2} \mathrm{O} \geq 14,0$.
\end{abstract}

keywords: feldspar, ceramic, indrustry minerals

INTRODUÇÃO Os pegmatitos de Coronel Murta são conhecidos devido à ocorrência de turmalina e berilo de qualidade gemológica além de peças-de-coleção (Pedrosa-Soares et al.1990, Pedrosa-Soares et al. 2001a). Cita-se como exemplo o Morro Redondo, um corpo pegmatítico ( $>50 \mathrm{~m}$ ) localizado a SW de Coronel Murta, que entre os anos de 1993 e 1995 produziu 11 toneladas de turmalinas coradas dos tipos verdelita e rubelita (Castañeda 1997).

Atualmente, o garimpo de pedras coradas, i.e. mineraisgema, constitui a principal atividade econômica do município, destacando a existência de inúmeras frentes de exploração abertas por garimpeiros de maneira rudimentar provocando extrações inadequadas. Por outro lado, existem poucas lavras para extração de minerais industriais no município, sendo o feldspato potássico o bem mineral mais extraído, devido principalmente ao seu grande volume de reservas e facilidade de extração. Entretanto, não há nenhum tipo de controle sistemático do feldspato extraído de corpos pegmatíticos da área estudada.

Apesar da significativa bibliografia que aborda condicio- nantes geológicos, mineralógicos, geoquímicos e estudos gemológicos dos pegmatitos, são escassos os estudos de minerais industriais e sua aplicação tecnológica. Portanto, a caracterização mineralógica, química e tecnológica dos feldspatos de Coronel Murta são pilares fundamentais para o desenvolvimento e potencialização da atividade mineraria da região, de forma concomitante à caracterização das lavras pegmatíticas. Cita-se, como exemplo a Lavra da Água Santa, que a partir da caracterização mineralógica e tecnológica dos feldspatos associada a um mapeamento de detalhe tornou-se possível o estabelecimento, por parte do PROGEMAS, de um plano de lavra para ampliação do final da galeria principal e da viabilidade da galeria superior.

Os objetivos deste trabalho foram: (a) determinar a viabilidade técnica de utilização do feldspato sódico-potássico de interesse, a microclina pertítica, do município de Coronel Murta como matéria-prima para diversos ramos da indústria, principalmente cerâmica e vidreira; (b) gerar um banco de dados qualificado com informações geológicas, geoambientais e de condições de condução da exploração dos pegmatitos de 21 lavras do município 


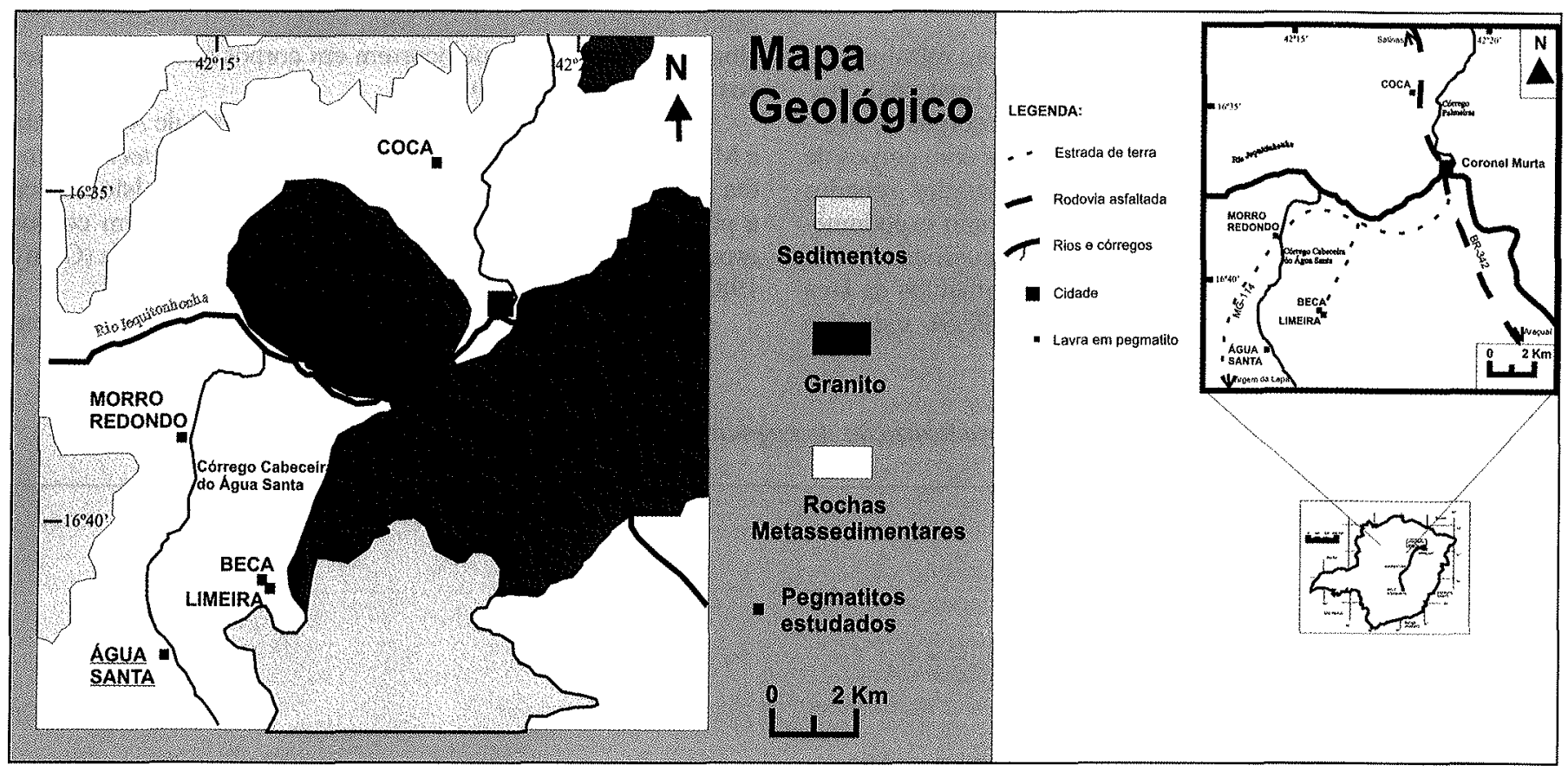

Figura 1-Esboço geológico da região do municipio de Coronel Murta em Minas Gerais e localização dos principais pegmatitos estudados.

de Coronel Murta; (c) fazer uma análise crítica dos parâmetros exigidos pelas indústrias vidreira e cerâmica para os feldspatos.

A área de estudo situa-se na região nordeste do Estado de Minas Gerais, no município de Coronel Murta, Médio Vale do Rio Jequitinhonha (Figura 1). A cidade de Coronel Murta está localizada a aproximadamente $60 \mathrm{~km}$ ao sul de Salinas e a $34 \mathrm{~km}$ ao norte de Araçuaí, dois dos principais municípios da região. A área de estudo enquadra-se inteiramente no mapa geográfico da Folha SE-23-X-B-VI Araçuaí, escala 1:100.000 (IBGE 1983) e Mapa Geológico da Folha Araçuaí, escala 1:100.000, Projeto Espinhaço (Pedrosa-Soares 1997), entre os paralelos $16^{\circ} 30^{\prime} \mathrm{e}$ $16^{\circ} 45^{\prime}$ e os meridianos $42^{\circ} 05^{\prime}$ e $42^{\circ} 20^{\prime}$, e corresponde a um quadrilátero de aproximadamente $700 \mathrm{~km}^{2}$ de superfície.

No contexto geológico, Coronel Murta localiza-se no Campo Pegmatítico de Coronel Murta-Virgem da Lapa, Distrito Pegmatítico de Araçuaí, parte integrante da Província Pegmatítica Oriental (Correia-Neves et al. 1986), que, em linhas gerais, corresponde à Província Gemológica Oriental do Brasil (PedrosaSoares et al. 2001). Os pegmatitos estudados foram originados a partir de resíduos magmáticos da cristalização de granitos do tipo $\mathrm{S}$, pertencentes à suíte $\mathrm{G} 4$ da Faixa Araçuaí (Pedrosa-Soares et al. 2001).

AMOSTRAGEM E METODOLOGIA Foram coletadas amostras de feldspato potássico das zonas mais internas (predominantemente a zona intermédia) dos pegmatitos das lavras estudadas. Estas amostras foram consideradas como sendo minérios destas lavras. A metodologia de estudo deste minério obedeceu as seguintes etapas:1) caracterização mineralógica macro e microscópica, ensaios cerâmicos (testes do botom e do azulejo) e avaliações industriais (teste visual de cor);2) Análises químicas de perda ao fogo e fluorescência de raios-X. Os parâmetros foram utilizados com base na indústria cerâmica de primeira qualidade, que admite os seguintes teores químicos: $\mathrm{SiO}_{2} \leq 67$; $\mathrm{TiO}_{2}<0,2 ; \mathrm{Al}_{2} \mathrm{O}_{3}>18,0 ; \mathrm{Fe}_{2} \mathrm{O}_{3} \leq 0,1 ; \mathrm{MgO} \leq 0,3 ; \mathrm{CaO} \leq 0,3 ;$ Perda ao Fogo $<0,5 ; \mathrm{Na}_{2} \mathrm{O}+\mathrm{K}_{2} \mathrm{O} \geq 13,0$. Entretanto, esta especificação química fica renegada a segundo plano em relação aos testes do botom e do azulejo, que determinam a qualidade cerâmica do minério feldspático.

Os ensaios cerâmicos de queima e piroexpansão realizados em amostras de feldspato potássico de zonas internas de pegmatitos foram executados nos fornos MVFLA da empresa LUFI Mineração e Beneficiamento Ltda. Os testes realizados foram do botom, para avaliar a cor, aumento de volume e porosidade do minério, e do azulejo, para determinação de sua transparência. A piroexpansão consiste em fundir a amostra de feldspato pulverizado e misturado ou não com frita na proporção de $2 / 3$ a uma temperatura de cerca de 1000 a $1100{ }^{\circ} \mathrm{C}$. Este processo permite identificar a cor da amostra a após a sua queima, determinar a variação de volume e a presença de bolhas no material pós-queima (grau de coesão).

CARACTERIZAÇ̃̃o dos PEgMATITOS DE CORONEL MURTA Foram caracterizadas vinte e uma (21) lavras pegmatíticas, seja para extração de minerais-gema ou peças-decoleção, seja para a produção de minerais industriais. Trata-se de pegmatitos simples a complexos encaixados em rochas metassedimentares (xisto, metagrauvaca, quartzito) das formações Salinas, Ribeirão da Folha e Chapada Acauã (Pedrosa-Soares 1997, Lima et al. 2002). Nenhum dos corpos encontra-se encaixado nos stocks de granito da suíte G4, localizados nos arredores da cidade de Coronel Murta.

Os corpos maiores são concordantes com a direção da xistosidade principal dos metamorfitos, geralmente N50E/40SE, mas nem sempre acompanham os seus mergulhos, o que corrobora as descrições de Pedrosa-Soares et al. (1990). O mergulho das superficies de encaixamento dos corpos pegmatíticos quase sempre é maior que o mergulho das encaixantes, variando de 70-80SE a vertical (Pinho-Tavares 2004). As formas dos pegmatitos são em geral lenticulares, mas também ocorrem corpos tabulares. Podem ser horizontais, como nas lavras do Alto da Copasa e Água Santa, ou verticais como na lavra do Juazeiro. Foram observadas feições estruturais de zonas acamadadas nos pegmatitos das lavras Beca, Limeira e Água Santa. Estas feições são caracterizadas por camadas decimétricas de feldspato potássico com intercrescimentos gráficos de quartzo intercaladas com 
camadas de textura média.

A grande maioria dos corpos pegmatíticos tem estrutura zonada, caracterizada por quatro zonas primárias (marginal, mural, intermediária e núcleo), cortadas ou não por corpos de substituição e preenchimentos de fratura. Pegmatitos com corpos de substituição de extensão métrica foram considerados zonados complexos (PZC), podendo conter minerais como espodumênio, ambligonita, cleavelandita e lepidolita. A ausência de turmalinas coradas (rosa, verde e/ou azul) nos pegmatitos da área é característica dos corpos zonados simples (PZS). Este fato ocorre porque as turmalinas rosa, verde e azul geralmente são elbaítas e Fe-elbaítas que ocorrem em corpos zonados complexos (Castañeda et al 2001).

Os dois maiores corpos caracterizados, lavras de Água Santa e Morro Redondo, estão distantes mais de $5 \mathrm{~km}$ do stock de biotita granito e constituem corpos zonados complexos. Isto ratifica a proposição de que pegmatitos deste tipo constituem corpos espessos ( $>20 \mathrm{~m}$ ) e situam-se distantes do granito-fonte (Cerný 1982, 1991a, Pedrosa-Soares et al. 1990).

A grande maioria dos garimpos estudados desenvolve-se em

Quadro 1 - Ficha cadastral de dados para caracterização de pegmatitos

1) NƯMERO de ordem nas tabelas 11 e 12.

2) MUNICÍPIO.

3) DISTRITO E/OU LOCALIDADE.

4) NOME do Pegmatito, Lavra Garimpeira, Serviço ou Mina.

5) LOCALIZAÇÃO dos pegmatitos em UTM, com GPS e altímetro.

6) TOPONÍMIA E CARACTERÍSTICAS LOCAIS, tais como acesso, relevo, drenagem, facilidades disponíveis como luz elétrica, água potável etc.

7) ESTÁGIO E TIPO DE EXPLORAÇ̃̃O (não explorado, exploração ativa ou inativa, a céu aberto ou subterrânea, garimpo ou mina em corpo único ou em agrupamento de corpos, etc).

8) HISTÓRICO DA PRODUÇÃO (minerais extraídos no passado e no presente, conforme informações obtidas no local).

\section{9) CARACTERIZAÇÃO GEOLÓGICA DO PEGMATITO:}

(a) Atitude do Corpo, obtida por meio de medidas de atitude das estruturas planares e lineares das rochas encaixantes, nas proximidades do contato com o pegmatito e afastado deste, além de medidas da atitude do pegmatito, de maneira a determinar a(s) atitude(s) do encaixamento do pegmatito pelas atitudes médias obtidas em cada parte do corpo.

(b) Forma do Corpo, obtida pela informação do tipo de rocha encaixante, análise dos afloramentos e suas atitudes, distribuição das zonas do pegmatito, etc.

(c) Tamanho do Corpo, determinado pela avaliação das dimensões entre capa e lapa (espessura), e entre topo e raiz. A classificação do corpo pegmatítico foi feita conforme a espessura entre capa e lapa nas seguintes categorias: $<0,5 \mathrm{~m}=$ muito pequeno; 0,5 a $5 \mathrm{~m}=$ pequeno; 5 a $15 \mathrm{~m}=$ médio; 15 a $50 \mathrm{~m}=$ grande; $>50 \mathrm{~m}=$ muito grande (Cameron et al. 1949).

(d) Estrutura Interna do Corpo, determinada por meio da observação de zonamento ou não, através da presença de corpos de substituição e preenchimentos de fratura, e pela classificação das zonas aflorantes (marginal, mural ou gráfica; intermediária; um ou mais núcleos) a partir das suas classes texturais, que são: cristais $<2,5 \mathrm{~cm}=$ fina ou granítica; cristais entre 2,5 a $10 \mathrm{~cm}=$ média; 10 a $30 \mathrm{~cm}=$ grossa $>30 \mathrm{~cm}=$ muito grossa (Cameron et al. 1949).

(e) Esboço do zonamento interno e modelo morfológico-estrutural do corpo, através de desenhos.

(f) Caracterização Mineralógica do Pegmatito, por determinação dos minerais essenciais e acessórios de cada unidade interna do pegmatito.

10) CONDIÇÕES DA LAVRA E SUGESTÕES, através do diagnóstico da situação dos túneis, cavas e frentes de lava.

\section{1) CARACTERIZAÇÃO GEOAMBIENTAL EXPEDITA.}

12) DOCUMENTÁRIO FOTOGRÁFICO do local, da rocha encaixante, do pegmatito, suas zonas e unidades tardias, de minerais extraídos e da mineralogia geral do corpo, e de estruturas ou texturas interessantes e/ou diferentes e/ou curiosas.

13) AMOSTRAGEM, com descrição do local da amostragem e a coordenada geográfica com GPS. Também se buscou coletar amostras de feldspato de rejeitos com potencial para uso industrial. 
Tabela 1 - Principais características das lavras em pegmatitos de Coronel Murta. As siglas utilizadas para os bens minerais são: qz $=q u a r t z o, f p=$ feldspato potássico, $a b=$ albita, $m s=$ muscovita, $t u=$ turmalina, $s c=$ schorlita, eb $=$ elbaita, be $=b e r i l o, m t=m o r-$

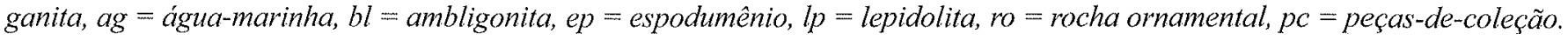

\begin{tabular}{|c|c|c|c|c|c|c|}
\hline $\mathbf{N}^{0}$ & Lavra & Bem Mineral & UTM $x$ & UTM y & Status & Local \\
\hline 1 & Juazeiro & $\mathrm{qz}, \mathrm{lp}, \mathrm{eb}, \mathrm{pc}, \mathrm{mt}$ & 799817 & 8160452 & ativa & Fazenda Rocinha \\
\hline 2 & Morro da Serra & $\mathrm{qz}, \mathrm{be}$ & 799400 & 8160644 & ativa & Fazenda Rocinha \\
\hline 3 & Alto da Copasa & $q z, f p, m s, b e, s c, a b$ & 800677 & 8161393 & ativa & Periferia da cidade \\
\hline 4 & Fazenda Boa Vista & $\mathrm{qz}, \mathrm{fp}, \mathrm{be}, \mathrm{eb}, \mathrm{pc}, \mathrm{ag}$ & 801201 & 8161888 & ativa & Fazenda Boa Vista \\
\hline 5 & Cido \& Fabio de Aires & $\mathrm{qz}, \mathrm{tu}, \mathrm{pc}$ & 800715 & 8162220 & ativa & Fazenda do Dr. Rubens \\
\hline 6 & Mario Alberto & $\mathrm{fp}, \mathrm{ms}$ & 800855 & 8162319 & desativada & Fazenda do Dr. Rubens \\
\hline 7 & Joaquim Barbosa & $\mathrm{fp}, \mathrm{ms}$, be & 801311 & 8162157 & desativada & Fazenda do Dr. Rubens \\
\hline 8 & Luiz de Zé Francisca & $q \mathrm{z}, \mathrm{fp}, \mathrm{ms}, \mathrm{pc}$ & 801290 & 8162244 & ativa & Faz. Nim de Miguel Pedro \\
\hline 9 & Joel Vieira Alves & $f p, m s, b e, p c, a b, e p$ & 799999 & 8162198 & ativa & Fazenda Palmeiras \\
\hline 10 & Valdivino P. Rodrigues & $\mathrm{qz}, \mathrm{fp}, \mathrm{pc}$ & 800405 & 8161880 & ativa & Fazenda Palmeiras \\
\hline 11 & Zé Matão & be, sc, qz & 800389 & 8162403 & ativa & Fazenda Palmeiras \\
\hline 12 & Miguel Pedro & $\mathrm{fp}, \mathrm{sc}$ & 800988 & 8162577 & ativa & Fazenda Palmeiras \\
\hline 13 & Coca & $\mathrm{qz}, \mathrm{fp}, \mathrm{be}$ & 799337 & 8163670 & desativada & Fazenda Palmeiras \\
\hline 14 & Beca & $f p$ & 794027 & 8154164 & ativa & Limeira \\
\hline 15 & Limeira & $\mathrm{fp}$ & 794150 & 8154047 & desativada & Limeira \\
\hline 16 & Água Santa & $q \mathrm{z}, \mathrm{fp}, \mathrm{be}, \mathrm{sc}, \mathrm{pc}$ & 792429 & 8151295 & ativa & Alto do Morro Redondo \\
\hline 17 & Morro Redondo & $\mathrm{fp}, \mathrm{q} z, \mathrm{lp}, \mathrm{bl}, \mathrm{eb}, \mathrm{ag}, \mathrm{pc}$ & 792220 & 8156750 & ativa & Morro Redondo \\
\hline 18 & Perobeiros & $q z, f p, b e$ & 799887 & 8161112 & ativa & Periferia da cidade \\
\hline 19 & Fazenda Turmalina & $q z, b e, f p, l p, b l, e b, p c$ & 800775 & 8166390 & ativa & Lorena \\
\hline 20 & Fazenda Cacimba & $q \mathrm{z}, \mathrm{fp}, \mathrm{ms}, \mathrm{tu}, \mathrm{pc}, \mathrm{be}$ & 799539 & 8160226 & ativa & Fazenda Cacimba \\
\hline 21 & Pedreira Granitos Colatina & ro, $f p$ & 805346 & 8171075 & ativa & Olho D'água \\
\hline
\end{tabular}

um único corpo. Entretanto, o serviço do Joel Vieira Alves atravessa dois pegmatitos, enquanto que a lavra do Miguel Pedro é executada em dois a quatro corpos pegmatíticos. O garimpo da Fazenda Boa Vista, que constitui mais de dez serviços distantes menos de $15 \mathrm{~m}$ entre si, é desenvolvido em um aglomerado de corpos pegmatíticos paralelos de espessura decimétrica a decamétrica (Pinho-Tavares et al. 2003).

Sistemática da Caracterização dos Corpos Pegmatíticos $\mathrm{A}$ caracterização de cada pegmatito foi realizada com base na ficha de dados desenvolvida neste trabalho (Quadro 1). Os dados coletados segundo a ficha são apresentados e resumidos, para cada uma das vinte e uma lavras, nas Tabelas 1 e 2 . As lavras encontram-se localizadas no mapa geológico (Figura 1) simplificado a partir de Lima et al. (2002).

\section{CARACTERIZAÇÃO DO FELDSPATO}

Química mineral A falta de conhecimento sobre as propriedades químicas e mineralógicas do material fundente (no caso da cerâmica e vidro, o feldspato potássico) representa fator complicador para a utilização do feldspato. As indústrias, principalmente do ramo das cerâmicas brancas, costumam ignorar o fato de o feldspato ser uma substância natural e como tal apresenta diferenças químicas ou mineralógicas de uma lavra para outra. $O$ percentual de cada óxido existente no mineral, mesmo presente em pequenas quantidades, determina a sua utilização. Nos processos cerâmicos, exercem a função de fundentes os óxidos de elementos al- calinos e alcalinos terrosos, como $\mathrm{K}_{2} \mathrm{O}, \mathrm{Na}_{2} \mathrm{O}, \mathrm{Li}_{2} \mathrm{O}, \mathrm{MgO} \mathrm{CaO}$, $\mathrm{BaO}$ e SrO; $\mathrm{o} \mathrm{Al}_{2} \mathrm{O}_{3}$ é estabilizante; $\mathrm{SiO}_{2}$ e $\mathrm{B}_{2} \mathrm{O}_{3}$ sã̃o vitrificantes; os óxidos dos metais de transição, e.g. $\mathrm{FeO}, \mathrm{Fe}_{2} \mathrm{O}_{3}, \mathrm{Fe}_{3} \mathrm{O}_{4}, \mathrm{MnO}$, $\mathrm{CuO}, \mathrm{CdO}, \mathrm{Ni}_{2} \mathrm{O}_{3}$, entre outros, são colorantes; e, por fim, são opacificantes principalmente $\mathrm{SnO}_{2}, \mathrm{TiO}_{2}$ e $\mathrm{ZrO}_{2}$ (comunicação escrita do Prof. Tito Tortori, Química Cerâmica).

A Tabela 3 apresenta os dados químicos dos feldspatos potássicos selecionados das lavras estudadas. A análise geral dos dados químicos do feldspato da Água Santa (AGF) mostra que a porção $\mathrm{SE}$ do pegmatito é mais rica em $\mathrm{SiO}_{2}, \mathrm{Na}_{2} \mathrm{O}$ e $\mathrm{Na}_{2} \mathrm{O}+$ $\mathrm{K}_{2} \mathrm{O}$, enquanto a parte mais a NW do corpo pegmatítico apresenta feldspato com maiores valores em $\mathrm{Al}_{2} \mathrm{O}_{3}$ e $\mathrm{Fe}_{2} \mathrm{O}_{3}$. Quanto aos segmentos industriais, pode-se dizer que segundo os dados de $\mathrm{SiO}_{2}$, e $\mathrm{Al}_{2} \mathrm{O}_{3}$ e $\mathrm{Fe}_{2} \mathrm{O}_{3}$ a porção $\mathrm{SE}$ é mais adequada para a produção de vidro $\mathrm{B}$, enquanto a zona intermédia interna a intermédia externa NW é adequada para cerâmica de $1^{\text {a }}$ qualidade.

Os teores de $\mathrm{Na}_{2} \mathrm{O}+\mathrm{K}_{2} \mathrm{O}$ mostram que todas os feldspatos potássicos do Beca (BE) enquadram-se nas especificações de vidro A e cerâmica de $1^{\text {a }}$ qualidade. Entretanto, levando-se em conta os dados dos óxidos isolados, apenas uma amostra atende às exigências da indústria cerâmica de $1^{\text {a }}$ qualidade, pois apresenta valor alto para $\mathrm{Na}_{2} \mathrm{O}$. Por outro lado, em relação a perda ao fogo (P.F.) nenhuma amostra pode ser considerada de boa qualidade para cerâmica de $1^{\text {a }}$ qualidade segundo especificações.

Todas as amostras do Coca (CC) apresentam teores de $\mathrm{Na}_{2} \mathrm{O}$ $+\mathrm{K}_{2} \mathrm{O}$ dentro das especificações da cerâmica de $1^{\text {a }}$ qualidade. 
Tabela 2 - Outras características das lavras em pegmatitos de Coronel Murta. Os tipos de pegmatitos são: PH = Pegmatito Homogeneo, $P S=$ Pegmatito Simples, PZS = Pegmatito Zonado Simples, PZC = Pegmatito Zonado Complexo (subtipos: PZC-lit = litinifero, $P Z C$-ab/ms = albita/muscovita, $P Z C$-ep = espodumênio).

\begin{tabular}{|c|c|c|c|c|c|c|}
\hline $\mathbf{N}^{\circ}$ & Lavra & Dimensão & Tipo & Forma & Atitude & $\begin{array}{c}\text { Rocha } \\
\text { Encaixante }\end{array}$ \\
\hline 1 & Juazeiro & grande a muito grande & PZC-lit & indefinida & $?$ & metagrauvaca \\
\hline 2 & Morro da Serra & muito grande & PZS & indefinida & $?$ & metagrauvaca \\
\hline 3 & Alto da Copasa & muito grande & $\mathrm{PZC}-\mathrm{ab} / \mathrm{ms}$ & tabular & $110 / 78$ e $105 / 90$ & xisto e metagrauvaca \\
\hline 4 & Fazenda Boa Vista & muito pequeno a grande & $\mathrm{PZS} / \mathrm{C}$ & tabular & $172 / 44$ e $314 / 75$ & xisto \\
\hline 5 & Cido \& Fabio de Aires & médio a grande & PZS & tabular & $080 / 90$ e $130 / 85$ & xisto \\
\hline 6 & Mario Alberto & grande a muito grande & $\mathrm{PZS}$ & tabular & $?$ & xisto \\
\hline 7 & Joaquim Barbosa & grande a muito grande & $\mathrm{PZS}$ & tabular & $147 / 68$ & xisto grauvaquiano \\
\hline 8 & Luiz de Zé Francisca & grande a muito grande & PZS & tabular & $122 / 79$ & xisto grauvaquiano \\
\hline 9 & Joel Vieira Alves & grande a muito grande & PZC-ep & indefinida & $046 / 60$ e $190 / 75$ & xisto \\
\hline 10 & Valdivino P. Rodrigues & muito grande & PZS & indefinida & $320 / 85$ e $356 / 75$ & xisto \\
\hline 11 & Zé Matão & médio a muito grande & $\mathrm{PH}$ & tabular & $164 / 72$ & xisto grauvaquiano \\
\hline 12 & Miguel Pedro & médio a grande & PZS & tabular & $282 / 81$ e $134 / 50$ & xisto \\
\hline 13 & Coca & muito grande & $\mathrm{PZS}$ & lenticular & $170 / 35$ e $197 / 42$ & xisto \\
\hline 14 & Beca & muito grande & PZS & tabular & $320 / 70$ & xisto \\
\hline 15 & Limeira & muito grande & $\mathrm{PZS}$ & tabular & $122 / 35$ e $115 / 55$ & xisto \\
\hline 16 & Água Santa & muito grande & $\mathrm{PZC}-\mathrm{ab} / \mathrm{ms}$ & tabular & $125 / 38$ e $175 / 46$ & xisto \\
\hline 17 & Morro Redondo & muito grande & PZC-lit & tabular & $060 / 80$ e $075 / 80$ & xisto e quartzito \\
\hline 18 & Perobeiros & muito grande & PZS & tabular & $010 / 80$ e $005 / 85$ & xisto \\
\hline 19 & Fazenda Turmalina & muito grande & PZC-lit & tabular & $038 / 70$ e $062 / 30$ & xisto e metagrauvaca \\
\hline 20 & Fazenda Cacimba & grande a muito grande & $\mathrm{PZC}-\mathrm{ab} / \mathrm{ms}$ & tabular & $175 / 85$ & xisto \\
\hline 21 & Pedreira Granitos Colatina & grande a muito grande & PH & cúpula & $?$ & $?$ \\
\hline
\end{tabular}

Apenas duas amostras apontam teores de álcalis acima do limite máximo admitido para vidro A. Por outro lado, de acordo com os parâmetros químicos de teores dos óxidos isolados apenas uma amostra está dentro das especificações para cerâmica de $1^{\text {a }}$ qualidade. Novamente, os valores de perda ao fogo (P.F.) são incompatíveis com os limites máximos admitidos pelo mercado de cerâmica de $1^{a}$ qualidade e vidro $A$.

Os feldspatos potássicos da lavra de Limeira (LM) atendem às exigências dos vidros $\mathrm{A}$ e $\mathrm{B}$ em relação aos conteúdos de $\mathrm{Na}_{2} \mathrm{O}+\mathrm{K}_{2} \mathrm{O}$, assim como, às especificações para álcalis da cerâmica de $1^{a}$ qualidade. De acordo com os parâmetros químicos de teores de óxidos isolados três das quatro amostras ajustam-se às especificações da indústria cerâmica de $1^{a}$ qualidade. Entretanto, sob o parâmetro de perda ao fogo todas as amostras excedem ao valor máximo de $0,5 \%$ de (P.F.) admitidos com máximos para cerâmica de $1^{\text {a }}$ qualidade e vidro A. Contudo, todas são adequadas para a produção de vidro B e cerâmica de $2^{a}$ qualidade. Apenas uma amostra apresenta valor de P.F. acima do $0,72 \%$ de $\mathrm{H}_{2} \mathrm{O}^{+}$da microclina micropertítica de pegmatito.

Os feldspatos potássicos das demais lavras estudadas, Juazeiro, Valdivino Pereira Rodrigues, Alto da Copasa, Fazenda Boa Vista, Luiz de Zé Francisca e Fazenda Turmalina enquadram-se segundos os padrões de $\mathrm{Na}_{2} \mathrm{O}+\mathrm{K}_{2} \mathrm{O}$ para vidro $\mathrm{A} e$ são compatíveis com a cerâmica de $1^{a}$ qualidade. Além disso, de acordo com os parâmetros químicos de teores de óxidos, sete amostras, dentre as 25 , estão adequadas à produção de cerâmica de $1^{\text {a }}$ qualidade.

Ensaios Cerâmicos Os ensaios cerâmicos consistem na quei$\mathrm{ma}, \mathrm{a} 1100^{\circ} \mathrm{C}$, de feldspato pulverizado a 200 mesh e misturado à Frita FMT-2331 da empresa Johnson Matthey Cerâmica Ltda., na proporção de $60 \%$ de frita para $40 \%$ de feldspato. O produto pós-queima é um botom de aproximadamente $2 \mathrm{~cm}$ de diâmetro. Um feldspato de boa qualidade para a indústria cerâmica, com este tipo de frita, não é expansível e apresenta-se branco pósqueima. A cor de queima branca é uma regra óbvia, uma vez que o produto final deve ser alguma variedade de cerâmica branca. O objetivo desse teste é avaliar a expansão e comparar a cor pósqueima com os botons-padrões.

No caso da empresa LUFI, o seu minério padrão (feldspato potássico e misturas) é produzido pela lavra Água Santa. Nenhuma das amostras coletadas durante o estudo apresentou piroexpansão, bem como a presença de bolhas ou poros em sua superfície. Em relação a cor pós-queima, dentre as setenta e duas amostras submetidas ao teste do botom apenas três foram reprovadas por apresentarem cor mais escura. Todas as outras foram consideradas de $1^{a}$ qualidade para indústria cerâmica, que adotam o botom com parâmetro qualificador.

O teste do azulejo é um refinamento do teste do botom para determinação de minérios destinados a indústria de produtos cerâmicos de $1^{a}$ qualidade, pois consiste em aplicar uma fina camada do minério feldspático puro (sem adição de frita) submetido a 
Tabela 3 - Análise quimica quantitativa por fluorescência de raios-X em feldspato potássico das lavras estudadas, incluindo os valores de perda ao fogo (PF). Análises realizadas no CPMTC-IGC-UFMG. Sigla das Lavras: AGF= Água Santa, BE=Beca, CC= Coca, $L M=$ Limeira, $M R=$ Morro Redondo, $C B=$ Fazenda Cacimba, $C M=$ Coronel Murta, JU = Juazeiro, MS= Morra da Serra,

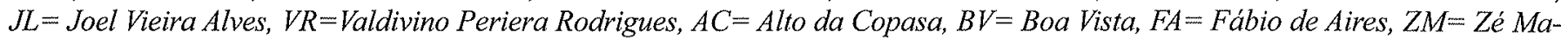
tão, $M P=$ Miguel Pedro, $M A=$ Mário Alberto, $Z F=$ Luiz de Zé Francisca, $T Q=$ Taquaral, $P B=$ Perobeiros, $T U=$ Turmalina.

\begin{tabular}{|c|c|c|c|c|c|c|c|c|c|c|c|c|c|c|c|}
\hline & \multirow[b]{2}{*}{ AGF $4 A$} & \multirow[b]{2}{*}{ AGF-4B } & \multirow[b]{2}{*}{ B AGF-4D } & & & & & & & & & \multicolumn{4}{|c|}{ ST-022 ST-024 ST-095 ST-096 } \\
\hline & & & & 4D AGF. & -3E AGF & F.4E BE- & $\mathrm{E}-1 \mathbf{A} \mathbf{B E}-$ ? & $2 \mathrm{~A}$ BE-3A & $A$ BE-4A & BE-5A & BE-6A & $\mathrm{CC}$ & $\mathrm{CC}$ & $\mathrm{CC}$ & $\mathrm{CC}$ \\
\hline $\mathrm{SiO}_{2}$ & 66,13 & 65,30 & \multicolumn{2}{|c|}{64,45} & 63,40 & $63,20 \quad 64$ & 64,60 & 66,10 & 64,50 & 64,30 & 64,70 & 64,80 & 64,30 & 64,00 & 63,40 \\
\hline $\mathrm{TiO}_{2}$ & 0,02 & 0,04 & \multicolumn{2}{|c|}{0,03} & 0,04 & 0,05 & 0,04 & 0,04 & 0,04 & 0,05 & 0,04 & 0,02 & 0,04 & 0,02 & 0,04 \\
\hline $\mathrm{Al}_{2} \mathrm{O}_{3}$ & 16,83 & 17,07 & 18, & 16 & 7,50 & $18,20 \quad 17$ & $17,90 \quad 16$ & $5,10 \quad 17,60$ & $50 \quad 16,70$ & 18,10 & 18,00 & 18,50 & 17,90 & 17,80 & 19,80 \\
\hline $\mathrm{Fe}_{2} \mathrm{O}_{3}(\mathrm{t})$ & 0,09 & 0,08 & & 12 & 0,24 & 0,18 & 0,13 & 0,27 & 0,14 & 0,13 & 0,12 & 0,04 & 0,08 & 0,08 & 0,09 \\
\hline MnO & 0,02 & 0,04 & &, 03 & 0,17 & 0,05 & 0,01 & 0,04 & 0,03 & 0,01 & 0,05 & 0,03 & $<0,01$ & 0,01 & 0,02 \\
\hline MgO & 0,04 & 0,03 & &, 02 & 0,01 & 0,03 & 0,02 &, 01 & 0,01 & 0,01 & 0,04 & 0,01 & 0,03 & 0,01 & 0,01 \\
\hline $\mathrm{CaO}$ & 0,12 & 0,11 & &, 22 & 0,12 & 0,12 & 0,14 &, 16 & 0,16 & 0,22 & 0,11 & 0,10 & 0,21 & 0,12 & 0,21 \\
\hline $\mathrm{Na}_{2} \mathrm{O}$ & 3,60 & 3,93 & & 17 & 3,11 & 3,45 & 3,45 & 3,58 & 3,54 & 3,53 & 3,60 & 3,33 & 3,21 & 3,51 & 2,89 \\
\hline $\mathrm{K}_{2} \mathrm{O}$ & 12,05 & 12,27 & 12, &, 48 & 3,80 & $12,90 \quad 12$ & $12,00 \quad 12$ & $2,30 \quad 12,10$ & $\begin{array}{ll}0 & 12,50\end{array}$ & 11,40 & 11,80 & 12,00 & 12,80 & 13,20 & 12,40 \\
\hline $\mathrm{Na}_{2} \mathrm{O}+\mathrm{K}_{2} \mathrm{O}$ & 15,65 & 16,19 & 15, & 65 & 6,91 & $16,35 \quad 15$ & $15,45 \quad 15$ & $5,58 \quad 15,68$ & $8 \quad 16,04$ & 14,93 & 15,40 & 15,33 & 16,01 & 16,71 & 15,29 \\
\hline PF & 0,64 & 0,60 & & 64 & 0,85 & 0,74 & 0,67 & 0,61 & 0,62 & 0,89 & 0,87 & 0,82 & 0,56 & 0,62 & 0,65 \\
\hline Total & 99,51 & 99,45 & 99,2 & 29 & 9,24 & $98,92 \quad 98$ & $98,29 \quad 98$ & $3,41 \quad 99,91$ & $1 \quad 97,62$ & $\quad 97,75$ & 98,46 & $6 \quad 98,83$ & 98,57 & 98,75 & 98,86 \\
\hline & $\begin{array}{c}\text { ST-016 S } \\
\text { LM }\end{array}$ & $\begin{array}{l}\text { ST-069 } \\
\text { LM }\end{array}$ & $\begin{array}{l}\text { T-097 S } \\
\text { LM }\end{array}$ & $\begin{array}{l}\text { ST-099 } \\
\text { LM }\end{array}$ & $\begin{array}{l}\text { ST-021 S } \\
\text { MR }\end{array}$ & $\begin{array}{l}\text { ST-071 S } \\
\text { MR }\end{array}$ & $\begin{array}{c}\mathrm{ST}-072 \\
\mathrm{MR}\end{array}$ & $\begin{array}{l}\text { ST-076 S1 } \\
\text { MR }\end{array}$ & $\begin{array}{l}\mathrm{T}-025 \mathrm{~S} \\
\mathrm{CB}\end{array}$ & $\begin{array}{l}\text { T-037 S } \\
\text { CM }\end{array}$ & $\begin{array}{l}\text { T'042 S } \\
\text { CM }\end{array}$ & $\underset{\mathrm{JU}}{\mathrm{ST}-078 \mathrm{~S}}$ & $\begin{array}{l}\text { ST-079 S } \\
\text { MS }\end{array}$ & $\begin{array}{c}\text { ST-080 S } \\
\text { JL }\end{array}$ & $\begin{array}{c}\text { ST-082 } \\
\text { VR }\end{array}$ \\
\hline $\mathrm{SiO}_{2}$ & 65,60 & 63,50 & 65,50 & 64,50 & 65,60 & 64,10 & 63,80 & 63,60 & 63,20 & 65,00 & 63,20 & 64,40 & 65,00 & 63,30 & 64,80 \\
\hline $\mathrm{TiO}_{2}$ & $0,02<c$ & 00,01 & 0,04 & 0,03 & 0,03 & 0,01 & 0,04 & 0,03 & 0,03 & 0,02 & 0,04 & 0,02 & 0,01 & 0,03 & 0,04 \\
\hline $\mathrm{Al}_{2} \mathrm{O}_{3}$ & 18,10 & 18,00 & 18,80 & 17,60 & 18,20 & 17,80 & 17,30 & 18,10 & 17,30 & 17,50 & 18,20 & 18,40 & 17,30 & 18,70 & 18,30 \\
\hline $\mathrm{Fe}_{2} \mathrm{O}_{3}$ & 0,05 & 0,08 & 0,01 & 0,05 & 0,05 & 0,12 & 0,09 & 0,12 & 0,06 & 0,12 & 0,12 & 0,05 & 0,03 & 0,08 & 0,19 \\
\hline $\mathrm{MnO}$ & 0,02 & $0,01<0$ & 0.01 & 0,02 & 0,02 & 0,01 & 0,02 & 0,01 & 0,01 & 0,02 & 0,01 & 0,02 & 0,01 & 0,03 & 0,01 \\
\hline $\mathrm{MgO}$ & 0,01 & 0,02 & 0,03 & 0,03 & 0,02 & 0,03 & 0,01 & 0,01 & 0,01 & 0,03 & 0,02 & 0,02 & 0,04 & 0,02 & 0,01 \\
\hline $\mathrm{CaO}$ & 0,12 & 0,11 & 0,23 & 0,11 & 0,09 & 0,12 & 0,22 & 0,14 & 0,12 & 0,16 & 0,14 & 0,16 & 0,21 & 0,13 & 0,12 \\
\hline $\mathrm{Na}_{2} \mathrm{O}$ & 3,20 & 3,17 & 3,32 & 3,36 & 3,60 & 3,59 & 3,21 & 2,93 & 3,48 & 3,26 & 3,11 & 2,73 & 2,84 & 2,69 & 3,10 \\
\hline $\mathrm{K} 2 \mathrm{O}$ & 10,80 & 13,80 & 11,70 & 12,60 & 11,60 & 12,80 & 13,90 & 14,20 & 13,60 & 11,90 & 13,60 & 13,00 & 13,50 & 13,80 & 11,70 \\
\hline $\mathrm{Na}_{2} \mathrm{O}+\mathrm{K}_{2} \mathrm{O}$ & 14,00 & 16,97 & 15,02 & 15,96 & 15,20 & 16,39 & 17,11 & 17,13 & 17,08 & 15,16 & 16,71 & 15,73 & 16,34 & 16,49 & 14,80 \\
\hline $\mathrm{PF}$ & 0,77 & 0,68 & 0,63 & 0,54 & 0,85 & 0,62 & 0,59 & 0,58 & 0,63 & 0,65 & 0,58 & 0,70 & 0,63 & 0,65 & 0,67 \\
\hline Total & 97,92 & 98,69 & 99,63 & 98,30 & 99,21 & $\mathbf{9 8 , 5 8}$ & 98,59 & 99,14 & 97,81 & 98,01 & 98,44 & 98,80 & 98,94 & 98,78 & 98,27 \\
\hline & $\begin{array}{c}\text { ST-084 } \\
\text { AC }\end{array}$ & $\begin{array}{l}\text { ST-085 } \\
\text { BV }\end{array}$ & $\begin{array}{c}\text { ST-086 } \\
\text { BV }\end{array}$ & $\begin{array}{c}\text { ST-088 } \\
\text { FA }\end{array}$ & $\begin{array}{c}\text { ST } \sim 090 \\
\text { ZM } \\
\end{array}$ & $\begin{array}{c}\text { ST.091 } \\
\text { MP }\end{array}$ & $\begin{array}{c}\text { ST } \sim 093 \\
\text { MA }\end{array}$ & $\begin{array}{c}\text { ST-094 S } \\
\text { ZF }\end{array}$ & $\begin{array}{l}\text { ST-100 } \\
\text { TQ }\end{array}$ & $\begin{array}{l}\text { ST-101 } \\
\text { TQ }\end{array}$ & $\begin{array}{c}\text { ST-103 } \\
\text { PB }\end{array}$ & $\begin{array}{c}\text { ST-106 } \\
\text { TU }\end{array}$ & $\begin{array}{c}\text { ST-110 } \\
\text { TU }\end{array}$ & $\begin{array}{c}\text { ST-112 } \\
\text { TU }\end{array}$ & \\
\hline $\mathrm{SiO}_{2}$ & 64,90 & 64,80 & 64,50 & 64,50 & 65,10 & 63,60 & 63,20 & 64,20 & 63,80 & 64,50 & 64,30 & 64,30 & 65,10 & 64,80 & \\
\hline $\mathrm{TiO}_{2}$ & 0,04 & 0,04 & 0,02 & 0,03 & 0,04 & 0,04 & 0,01 & 0,02 & 0,01 & 0,04 & 0,03 & 0,04 & 0,02 & 0,03 & \\
\hline $\mathrm{Al}_{2} \mathrm{O}_{3}$ & 18,40 & 17,60 & 18,30 & 18,10 & 16,80 & 18,10 & 17,90 & 18,10 & 17,80 & 17,80 & 17,80 & 18,30 & 18,70 & 19,60 & \\
\hline $\mathrm{Fe}_{2} \mathrm{O}_{3}$ & 0,21 & 0,12 & 0,05 & 0,02 & 0,05 & 0,13 & 0,08 & 0,01 & 0,02 & 0,06 & 0,05 & 0,12 & 0,14 & 0,09 & \\
\hline $\mathrm{MnO}$ & 0,02 & 0,01 & 0,02 & 0,01 & 0,02 & 0,01 & 0,04 & 0,01 & 0,01 & 0,02 & 0,01 & 0,30 & 0,01 & 0,02 & \\
\hline $\mathrm{MgO}$ & 0,03 & 0,02 & 0,01 & 0,02 & 0,01 & 0,02 & 0,02 & 0,01 & 0,02 & 0,03 & 0,20 & 0,01 & 0,03 & 0,01 & \\
\hline $\mathrm{CaO}$ & 0,15 & 0,14 & 0,07 & 0,09 & 0,14 & 0,15 & 0,88 & 0,05 & 0,14 & 0,12 & 0,14 & 0,18 & 0,15 & 0,12 & \\
\hline $\mathrm{Na}_{2} \mathrm{O}$ & 3,40 & 3,13 & 2,49 & 2,98 & 3,15 & 2,25 & 2,63 & 3,30 & 3,87 & 3,38 & 3,31 & 3,27 & 1,85 & 4,03 & \\
\hline $\mathrm{K}_{2} \mathrm{O}$ & 12,10 & 11,60 & 13,20 & 12,40 & 13,80 & 13,80 & 13,70 & 12,30 & 13,40 & 12,80 & 12,50 & 11,80 & 12,10 & 10,60 & \\
\hline $\mathrm{Na} 2 \mathrm{O}+\mathrm{K}_{2} \mathrm{O}$ & 15,50 & 14,73 & 15,69 & 15,38 & 16,95 & 16,05 & 16,33 & 15,60 & 17,27 & 16,18 & 15,81 & 15,07 & 13,95 & 14,63 & \\
\hline PF & 0,61 & 0,58 & 0,45 & 0,63 & 0,48 & 0,45 & 0,54 & 0,68 & 0,51 & 0,60 & 0,52 & 0,59 & 0,49 & 0,58 & \\
\hline Total & 99,25 & 97,46 & 98,66 & 98,15 & 99,11 & 98,10 & 98,46 & $\mathbf{9 8 , 0 0}$ & 99,07 & 98,75 & 98,34 & 98,32 & 98,10 & 99,30 & \\
\hline
\end{tabular}

queima sobre uma placa de azulejo para verificar a sua transparência, propriedade considerada indesejável para a indústria de cerâmica branca. Os procedimentos para a preparação da amostra e da queima são os mesmos adotados para confecção do botom. Neste teste do azulejo, o minério que queima transparente é des- cartado, mesmo se já tiver sido aprovado no teste do botom.

Foram submetidas ao teste do azulejo algumas amostras de feldspato das lavras de Água Santa e Beca. Todas as amostras apresentaram cor de queima branca, entretanto três amostras da Água Santa mostraram-se transparentes após a queima sendo 
reprovadas no teste do azulejo e consideradas inadequadas para a produção de cerâmica de $1^{a}$ qualidade.

CONCLUSÕES A análise sistemática das lavras pegmatíticas de Coronel Murta permitiu apontar que a chance de ter ortoclásio, um dos principais minerais industrial, é maior para um pegmatito interno ou marginal do que para um pegmatito externo e distante do granito-fonte. Ao mesmo tempo, a avaliação paragenética dos pegmatitos mostra que as zonas externas (mural e marginal) de corpos zonados simples e complexos apresentam composição mineralógica bastante similar. Em ambos os tipos de pegmatitos, a zona marginal é composta por quartzo, albita, muscovita, feldspato potássico e pouca schorlita. Já a zona mural mostra porções de intercrescimentos gráficos de quartzo em feldspato potássico e outras porções com feldspato potássico, quartzo, muscovita, albita e schorlita.

Em relação ao uso dos feldspatos para a industria, de acordo com as especificações existentes não se pode afirmar que os minérios destinados à indústria vidreira são de pior qualidade que aqueles utilizados pela indústria cerâmica branca, ou sequer de melhor qualidade que os destinados à fabricação de eletrodos para solda. $O$ que se observa é que as indústrias cerâmica e vidreira são mais exigentes quanto às especificações químicas dos feldspatos. Na indústria cerâmica, apesar de ser considerada a composição química, na prática são realizados ensaios de fusibilidade, observando-se, por exemplo, o escorrimento (fluidez), "fervura" (escape de gases formando bolhas, devido à presença de minerais hidratados), cor etc. No entanto, algumas empresas podem ter especificações químicas e outros parâmetros particulares, mas que não são adotados generalizadamente. Neste sentido, os resultados dos ensaios cerâmicos mostraram que setenta e uma das setenta e duas amostras submetidas ao teste do botom foram consideradas minérios de primeira qualidade para a indústria cerâmica, enquanto o teste do azulejo aprovou nove das doze amostras avaliadas, comprovando que os padrões das especificações químicas exigidos pela industria pode ter maior flexibilidade. Ainda neste contexto, a exploração de pegmatitos homogêneos e corpos com porções de textura gráfica deveriam ser voltado para o fim ornamental, apesar de a retirada do feldspato potássico puro não ser um procedimento complicado nestes casos. Atualmente as rochas ornamentais de textura pegmatítica têm grande valorização no mercado.

Agradecimentos Os autores agradecem o suporte financeiro dos seguintes projetos: (1) "Exploração de Pegmatitos Portadores de Gemas: Uma Abordagem Inovadora", coordenado pela Profa. Maria José Gazzi Salum (Escola de Engenharia-UFMG) e financiado pelo CTMineral/CNPq; (2) "PROGEMAS MG", coordenado pela Profa. Maria José Gazzi Salum (Escola de Engenharia-UFMG) e financiado pelo Fundo Verde Amarelo do CTMineral/FINEP; e (3) "Evolução e Recursos Minerais do Orógeno Araçuar", coordenado pelo Prof. Antônio Carlos Pedrosa-Soares (CPMTC-IGC-UFMG) e financiado pelo CNPq. Além destes projetos, à CAPES, pela concessão de bolsa de mestrado ao primeiro autor e pelo Projeto PRODOC/CAPES. Também deve ser mencionado o apoio fornecido pela empresa LUFI Mineração e Beneficiamento Ltda., desde a hospitalidade com a qual nos recebeu em Coronel Murta até ao suporte laboratorial nas análises dos minérios feldspáticos para a elaboração da mencionada dissertação de mestrado.

\section{Referências}

Castañeda C. 1997. Caracterização mineralógica de turmalinas do Distrito Pegmatítico de Araçuaí. Dissertação de Mestrado, UFOP, Ouro Preto, $146 \mathrm{p}$.

Castañeda C., César-Mendes J., Pedrosa-Soares, A.C. 2001. Turmalina. In: C. Castañeda, J. E. Addad, A. Liccardo (eds.) Gemas de Minas Gerais. SBG-MG, Belo Horizonte, pp.: 152-180.

Cerný P. 1982. Anatomy and classification of granitic pegmatites. Min. Assoc. Canada, Short Course, Handbook 8.

Cerný P. 1991. Rare-element granitic pegmatites. Part I: Anatomy and internal evolution of pegmatitic deposits. Geoscience Canada, 18(2):49-67

Correia-Neves J.M., Pedrosa-Soares A.C., Marciano V.R.P.R.O. 1986. A Província Pegmatítica Oriental do Brasil à luz dos conhecimentos atuais. Rev. Bras. Geoc., 16(1):106-118.

Lima S.A.A., Martins-Neto M.A., Pedrosa-Soares A, C., Cordani U., Nutman A. 2002. A Formação Salinas na área-tipo, NE de Minas Gerais: uma proposta de revisão da estratigrafia da Faixa Araçuaí com base em evidências sedimentares, metamórficas e idades U-Pb SHRIMP. Rev. Bras. Geoc., 32(4):491-500.
Pedrosa-Soares A. C. 1997. Geologia da Folha Araçuaí. In: J.H. Grossi-Sad, L.M. Lobato, A.C.Pedrosa-Soares, B.S Soares-Filho. (eds.) Projeto Espinhaço. COMIG - Companhia Mineradora de Minas Gerais, Belo Horizonte, CD-ROM, p. 715-852.

Pedrosa-Soares A.C., Correia-Neves J.M., Leonardos O.H. 1990. Tipologia dos pegmatitos de Coronel Murta - Virgem da Lapa, Médio Jequitinhonha, Minas Gerais. Revista Escola de Minas, 43(4):4454.

Pedrosa-Soares A.C., Pinto C.P., Netto C., Araujo M.C., Castañeda C., Achtschin A.B., Basílio M.S. 2001. A Província Gemológica Oriental do Brasil. In: C. Castañeda, J.E. Addad, A. Liccardo (eds.) Gemas de Minas Gerais.: SBG-MG, Belo Horizonte, pp.: 16-33.

Pinho-Tavares S. 2004. O feldspato industrial de Coronel Murta, $M G$ : caracterização de lavras em pegmatitos e do feldspato potássico na perspectiva de aplicações à industria cerâmica e vidreira. Dissertação de Mestrado, IGC-UFMG, Belo Horinzonte, 241 p.

Manuscrito A-1595

Revisão aceita em 26 de setembro de 2006 